\title{
Parameterization of a Battery Simulation Model Using Numerical Optimization Methods
}

\author{
Robyn A. Jackey \\ The MathWorks, Inc. \\ Gregory L. Plett \\ University of Colorado at Colorado Springs \\ Martin J. Klein \\ Compact Power Inc.
}

Copyright (C) 2009 SAE International

\begin{abstract}
Typically, battery models are complex and difficult to parameterize to match real-world data. Achieving a good generalized fit between measured and simulated results should be done using a variety of laboratory data. Numerical optimizations can ensure the best possible fit between a simulation model and measured data, given a set of constraints.

In this paper, we propose a semi-automated process for parameterizing a lithium polymer battery (LiPB) cell simulation model that is able to satisfy constraints on the optimized parameters. This process uses a number of measured data sets under a variety of conditions. An iterative numerical optimization algorithm using Simulink Parameter Estimation was implemented to estimate parameter values by minimizing error between measured and simulated results.
\end{abstract}

\section{INTRODUCTION}

For many battery-based systems, it is necessary to be able to accurately estimate values indicative of internal states and parameters of the electrochemical cells themselves. Example systems include electric vehicles (EVs) and hybrid electric vehicles (HEVs). In both of these applications, we desire estimates of battery stateof-charge (SOC) and battery state-of-health $(\mathrm{SOH})$.
These estimates are often obtained by using models, a method that further necessitates an accurate mathematical description (model) of the dynamic characteristics of the cells. In this paper, we first describe a suitable model structure and then discuss how the parameters of this model may be automatically fit using MATLAB, Simulink, and Simulink Parameter Estimation.

For a battery model to be useful, it should contain as identifiable parameters those values that are to be estimated. For example, if we wish to estimate SOC, then SOC must be a variable in the equations of the model. We may also wish to estimate cell resistance and capacity, both of which contribute to a description of the cell's SOH. If so, these values should also appear as variables in the model.

The most accurate estimators of cell $\mathrm{SOC}$ and $\mathrm{SOH}$ that we are aware of are based on Kalman filtering methods ${ }^{[1-4]}$. Kalman filters impose further restrictions on the form that the model equations must take. In particular, the model must be in a "state-space" form:

$$
\begin{aligned}
x_{k+1} & =f\left(x_{k}, u_{k}, w_{k}\right) \\
y_{k} & =g\left(x_{k}, u_{k}, v_{k}\right)
\end{aligned}
$$

where $x_{k}$ is the state vector at discrete-time index $k, u_{k}$ is the measured system input vector at time $\mathrm{k}$ (perhaps

\footnotetext{
The Engineering Meetings Board has approved this paper for publication. It has successfully completed SAE's peer review process under the supervision of the session organizer. This process requires a minimum of three (3) reviews by industry experts.

All rights reserved. No part of this publication may be reproduced, stored in a retrieval system, or transmitted, in any form or by any means, electronic, mechanical, photocopying, recording, or otherwise, without the prior written permission of SAE.

ISSN 0148-7191

Positions and opinions advanced in this paper are those of the author(s) and not necessarily those of SAE. The author is solely responsible for the content of the paper.

SAE Customer Service: Tel: $\quad 877-606-7323$ (inside USA and Canada)

Tel: $\quad$ 724-776-4970 (outside USA)

Fax: $\quad 724-776-0790$

Email: CustomerService@sae.org

SAE Web Address: $\quad$ http://www.sae.org
} 
including battery-pack current, temperature, etc.), and $\mathrm{w}_{\mathrm{k}}$ is unmeasured "process noise" (modeling inaccuracy of the cell model). The system output is $y_{k}$, and $v_{k}$ models sensor noise. The stochastic inputs $w_{k}$ and $v_{k}$ are assumed to be zero-mean white Gaussian random processes with covariance matrices $\Sigma_{\mathrm{w}}$ and $\Sigma_{\mathrm{v}}$, respectively. Equation (1) is called the "state equation," (2) is called the "output equation," and $f()$ and $g()$ are (possibly nonlinear) functions, specified by the particular cell model used.

To be more specific, the system input vector $u_{k}$ typically contains the instantaneous cell current $\mathrm{i}_{\mathrm{k}}$. It may also contain the cell temperature $T_{k}$, an estimate of the cell's nominal capacity $C_{k}$, and/or an estimate of the cell's internal resistance $R_{k}$, for example. The system output is typically a scalar but may be vector valued as well. Here we consider the output to be the cell's loaded terminal voltage-not at-rest open-circuit-voltage (OCV). The system's state vector $x_{k}$ in some way represents in summary form the total effect of all past input to the system so that the present output may be predicted solely as a function of the state and present input. Values of past inputs are not required.

\section{ENHANCED SELF-CORRECTING CELL MODEL}

The model that we use in this paper is a specific variant of what has been called the "enhanced self-correcting" (ESC) cell model ${ }^{[1-4]}$. We briefly review the model equations in this section. The final form of the model fits within the framework of equations (1) and (2), so it may be used for estimating $\mathrm{SOC}$ and $\mathrm{SOH}$ using Kalman filter techniques.

The basis for the SOC state equation is developed as follows: If $z(t)=S O C$ at time $t$, we know that

$$
\mathrm{z}(\mathrm{t})=\mathrm{z}(0)-\int_{0}^{\mathrm{t}} \frac{\eta(\mathrm{i}(\tau)) \mathrm{i}(\tau)}{\mathrm{C}_{\mathrm{n}}} \mathrm{d} \tau,
$$

where $C_{n}$ is the nominal capacity of the cell, $i(t)$ is the cell current at time $\mathrm{t}$, and $\eta(\mathrm{i}(\mathrm{t}))$ is the Coulombic efficiency of the cell. A discrete-time approximate recurrence may then be written as

$$
\mathrm{z}_{\mathrm{k}+1}=\mathrm{z}_{\mathrm{k}}-\frac{\eta\left(\mathrm{i}_{\mathrm{k}}\right) \mathrm{i}_{\mathrm{k}} \Delta \mathrm{t}}{\mathrm{C}_{\mathrm{n}}},
$$

where $\Delta t$ is the sampling period (in hours). Equation (4) is used to include SOC in the state vector of the cell model as it is in state equation format already, with SOC as the state and $i_{k}$ as the input.

The dynamics of the change of polarization voltage are also captured by a state equation. We add "filter states" with linear dynamics:

$$
\left[\mathrm{f}_{\mathrm{k}+1}\right]=[\operatorname{diag}(\alpha)]\left[\mathrm{f}_{\mathrm{k}}\right]+\mathrm{i}_{\mathrm{k}} .
$$

The vector $\alpha$ has $\mathrm{N}$ filter "poles," with $|\alpha|<1$ for stability, corresponding to time constants of the polarization voltage dynamics. We use $\mathrm{N}=2$.

A further phenomenon captured by a state equation is hysteresis. A cell that has recently undergone a charge event will have a higher rest voltage than one that has undergone a discharge event, even at the same SOC. That is, voltage does not decay to OCV, but to OCV plus or minus a factor based on the hysteresis of the cell. We note that hysteresis is not a phenomenon generally associated with lithium-ion systems, since most applications have been in the light portable electronics area where SOC accuracy is not as critical as in the HEV application and where temperatures are not as extreme. It is, however, very pronounced at low temperatures and can lead to SOC errors as large as $\pm 40 \%$ if the estimate is based simply on OCV (even with full cell relaxation).

A hysteresis state implementing a linear-time-varying difference equation may be modeled as:

$$
\begin{aligned}
h_{k+1}= & \exp \left(-\left|\frac{\eta\left(i_{k}\right) i_{k} \gamma \Delta t}{C_{n}}\right|\right) h_{k}+ \\
& \left(1-\exp \left(-\left|\frac{\eta\left(i_{k}\right) i_{k} \gamma \Delta t}{C_{n}}\right|\right)\right) \operatorname{sgn}\left(i_{k}\right) .
\end{aligned}
$$

where the sgn function returns -1 if its argument is negative, 0 if its argument is zero, and +1 if its argument is positive and where $\gamma$ is a hysteresis rate constant.

The three components of the system state are combined:

$$
x_{k}=\left[\begin{array}{lll}
f_{k}^{\top} & h_{k} & z_{k}
\end{array}\right]^{\top} .
$$

The corresponding equations for $f_{k}, h_{k}$, and $z_{k}$ also combine to form the vector function $f()$.

The cell terminal voltage is modeled by the output equation $\mathrm{g}(\mathrm{)}$. With the states of the system as defined, the ESC model computes:

$$
y_{k}=O C V\left(z_{k}\right)+C\left[f_{k}\right]-R_{k}+M h_{k} .
$$

The voltage is computed as the sum of the open-circuitvoltage at the present SOC, plus a weighted sum of the polarization voltage states, minus ohmic losses, plus hysteresis. ( $\mathrm{M}$ represents the maximum hysteresis voltage at the present temperature.)

A further constraint on equation (7) is that during a constant-current charge or discharge, the polarization filter voltages must converge to zero so that $y_{k} \rightarrow O C V(S O C)-I \times R$ (plus hysteresis) [4, Part 2]. All components $C_{k}$ of the vector $C$ are free to be optimized except the Nth, which is computed as: 


$$
\mathrm{C}_{\mathrm{N}}=-\sum_{\mathrm{k}=1}^{\mathrm{N}-1} \mathrm{C}_{\mathrm{k}} \frac{\left(1-\alpha_{\mathrm{N}}\right)}{\left(1-\alpha_{\mathrm{k}}\right)}
$$

\section{IMPLEMENTING THE ESC MODEL}

The mathematical equations that define the ESC model have now been presented, but numeric values are needed for the constants to complete the model. In particular, we require values for:

- A Coulombic efficiency factor $\eta(\mathrm{i}(\mathrm{t}))$. The highefficiency LiPB cells for which we report results have Coulombic efficiency that is indistinguishable from unity, so we use a constant value of 1.0.

- A nominal cell capacity, $C_{n}$. These cells are approximately 5 Ah cells, but our method is able to identify the exact value based on experimental data, as noted in following sections.

- A vector of filter poles, $\alpha$. We use two filter poles, or $\mathrm{N}=2$. We find that the system has one slow filter pole that is insensitive to temperature variation, so we fix its value at 0.99999 . A second filter pole is found by optimizing $\beta$ and computing the pole location to be $0.99998 \tanh (\beta)$. The tanh function ensures that the resulting filter pole will be constrained to have magnitude less than unity, and result in a stable filter.

- A hysteresis rate constant, $\gamma$. The simulations show that the model is relatively insensitive to $\gamma$ within a wide range. We choose $\gamma$ such that $\gamma \Delta t=1$.

- A vector of polarization voltage blending factors, C.

- A maximum hysteresis voltage, M.

- An ohmic resistance, R.

- A function that computes OCV as a function of SOC.

Note that the remaining unknown parameters $C_{n}, \beta, C$, $M, R$, and the OCV function may all be temperature dependent.

Figure 1 shows a Simulink diagram of a subsystem that implements the state equation of the ESC model. There are four inputs: the cell current $i_{k}$, which is input port "Current"; the first polarization voltage filter pole $\alpha_{1}$, which is input "a1"; the second polarization voltage filter pole $\alpha_{2}$, which is input "a2"; and the cell capacity $\mathrm{C}_{\mathrm{n}}$, which is input port "Capacity." The subsystem computes updated values of the cell state: the cell SOC $z_{k}$, which is output port "SOC"; the cell hysteresis voltage value $h_{k}$, which is output port "hyst"; the first polarization voltage state $f_{k, 1}$, which is output port "f1"; and the second polarization voltage state $f_{k, 2}$, which is output port "f2." The only discrepancy between this diagram and the prior equations is the "Gain factor" of 1e-4, which is used to scale the polarization voltage states to roughly the same amplitude range as the SOC and hysteresis states. The optimization process for the model automatically compensates by computing "C" vector parameters that are correspondingly larger than if the gain factor were absent.

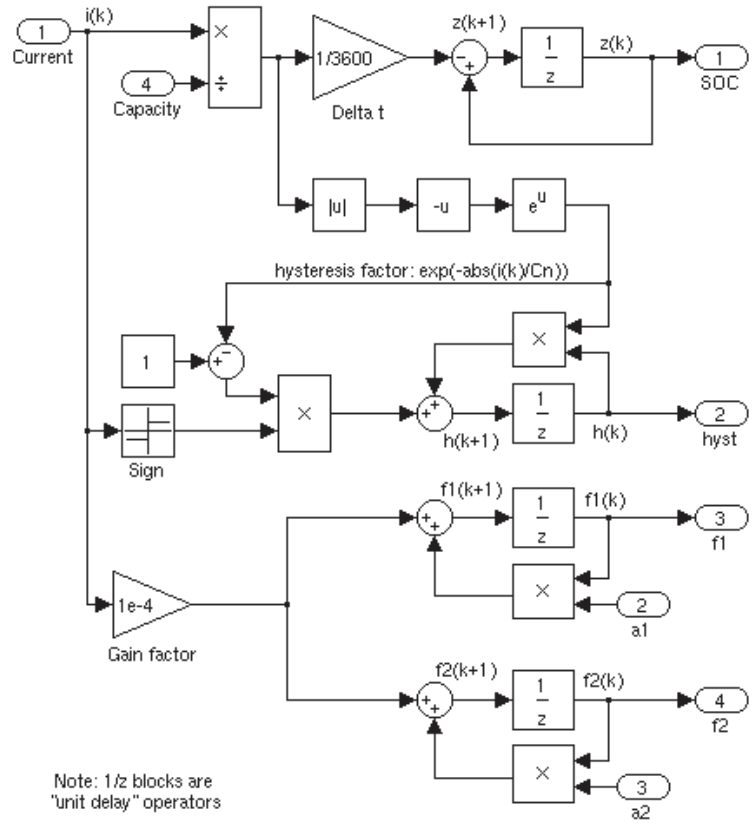

Figure 1. Simulink diagram of a subsystem that implements the state equation of the ESC battery cell model.

Figure 2 shows a Simulink diagram of a subsystem that implements the output equation of the ESC model. There are 10 inputs: the cell current $i_{k}$, which is input port "Current"; the cell temperature, which is input port "Temperature"; the cell SOC $z_{k}$, which is input "SOC"; the cell hysteresis value $h_{k}$, which is input "hyst"; the first polarization voltage $f_{k, 1}$, which is input " $f 1$ "; the second polarization voltage $\mathrm{f}_{\mathrm{k}_{2}}$, which is input " $\mathrm{f} 2$ "; the cell resistance $\mathrm{R}$, which is input "Resistance"; the cell maximum hysteresis level $M$, which is input "MaxHyst"; the first $\mathrm{C}$ vector component $\mathrm{C}_{1}$, which is input "c1"; and the second $\mathrm{C}$ vector component $\mathrm{C}_{2}$, which is input "c2." The subsystem first uses temperature and $\mathrm{SOC}$ with lookup tables to compute OCV from SOC at the present temperature (using a method described in the following section). Then, the output equation of the ESC model is computed based on all inputs. 


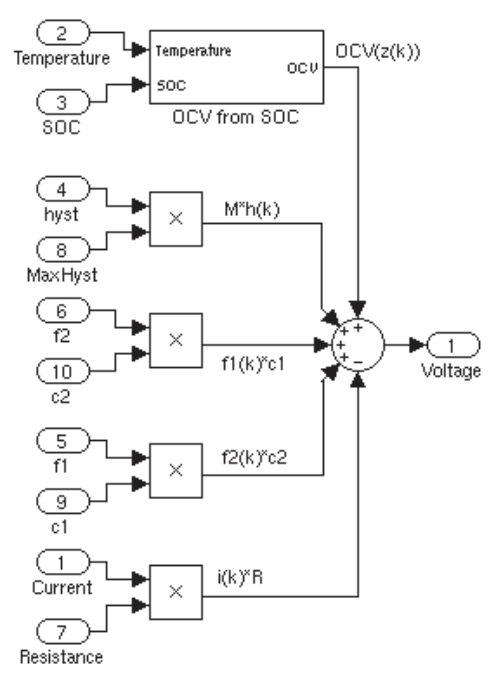

Figure 2. Simulink diagram of a subsystem that implements the output equation of the ESC battery cell model.

\section{DATA COLLECTION AND BASIC RESULTS}

Data from cell tests was collected for the purpose of fitting parameter values to the ESC cell model. Two sets of tests were performed. One recorded cell responses that could be used to compute an OCV versus SOC relationship at different temperatures, and the second recorded cell responses that could be used to determine the dynamic parameters of the cell model. This section describes these two types of test.

The data was gathered from prototype LiPB cells. These cells comprise a $\mathrm{LiMn}_{2} \mathrm{O}_{4}$ cathode and an artificial graphite anode, have a nominal capacity of $5 \mathrm{Ah}$ and a nominal voltage of $3.8 \mathrm{~V}$, and are designed for highpower applications. For the tests, we used a Tenney thermal chamber and an Arbin BT2000 cell cycler. Each channel of the Arbin was capable of $20 \mathrm{~A}$ current, and multiple channels were connected in parallel to achieve higher current levels. The cycler's voltage measurement accuracy was $\pm 5 \mathrm{mV}$, and its current measurement accuracy was $\pm 200 \mathrm{~mA}$. Cell variability, even with these prototype (handmade) cells, was small, and partly mitigated by wiring several cells in parallel during testing to electrically average the dynamic behavior. We expect the results from testing these cells to be representative of what we would see in production cells.

OCV VERSUS SOC - The first set of cell tests was done to evaluate the OCV versus SOC relationship at different temperatures. Three cells were connected in parallel to electrically average their responses. The tests comprised fully charging the cells at room temperature, allowing the cells to soak at the target temperature for two hours, discharging the cells at a $\mathrm{C} / 30$ rate until fully discharged, and charging the cells at a $\mathrm{C} / 30$ rate until fully charged. Tests were conducted at nine temperatures spanning the operating temperature range of these cells, from $-30^{\circ} \mathrm{C}$ to $50^{\circ} \mathrm{C}$.
The test data was analyzed by first computing a voltage relationship versus SOC for the discharge portion of the test, and then computing a second voltage relationship versus SOC for the charge portion of the test. At each SOC, the OCV was computed to be the average of the discharge voltage and the charge voltage. This process greatly diminishes the contributions of ohmic voltage and hysteresis voltage from the computed OCV relationship.

This analysis produces an individual OCV versus $\mathrm{SOC}$ relationship for each temperature. A large twodimensional table could be constructed to store this information and be used for computing OCV versus SOC at different temperatures, but the regular structure of the data suggested a better relationship. We model OCV as a function of SOC and temperature as follows:

$$
\operatorname{OCV}\left(z_{k}, t_{k}\right)=\operatorname{OCV}\left(z_{k}, 0\right)+t_{k} \Delta \operatorname{OCV}\left(z_{k}\right)
$$

That is, the open-circuit-voltage at any given SOC level and temperature was computed as the OCV at that SOC level at $0^{\circ} \mathrm{C}$ plus the temperature times a temperaturecorrection factor at that SOC level. This allows the OCV at any SOC and temperature to be computed using two one-dimensional table lookups versus a two-dimensional table lookup: a savings in storage and ultimately in computation.

Figure 3 displays the processed OCV versus SOC relationships for three temperatures in the operating range of the cell: minimum temperature, room temperature, and maximum temperature.

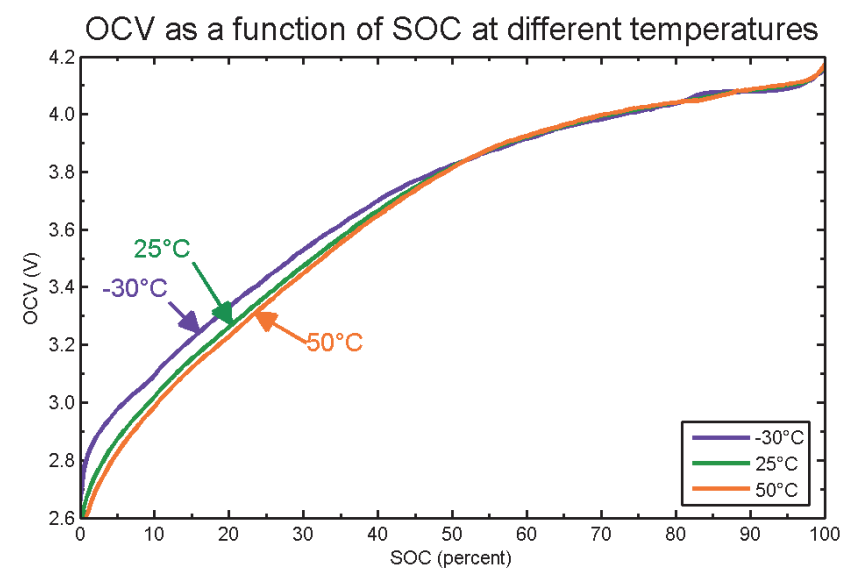

Figure 3. OCV test: OCV versus SOC over the operating range of the cell.

DYNAMIC CELL PARAMETERS - The second test was a sequence of 19 "urban dynamometer driving schedule" (UDDS) cycles, separated by $15 \mathrm{~A}$ discharge pulses and five-minute rests, and spread over the entire $\mathrm{SOC}$ range. Rate as a function of time for one of the UDDS cycles is plotted in Figure 4. SOC as a function of time is plotted in Figure 5. The cell starts fully charged and is immediately discharged to less than $90 \%$ to bring it within its normal operating range. The test continues from there. We see that SOC increases by about $5 \%$ 
during each UDDS cycle but is brought down about $7 \%$ during each discharge between cycles. The entire operating range for these cells ( $10 \%$ SOC to $90 \%$ SOC) is excited during the cell test. This dynamic data was used to identify the remaining parameters of the cell model, as described in the next sections. The goal is to have the cell model output resemble the cell terminal voltage under load as closely as possible, at all times, when the cell model input is equal to the cell current.

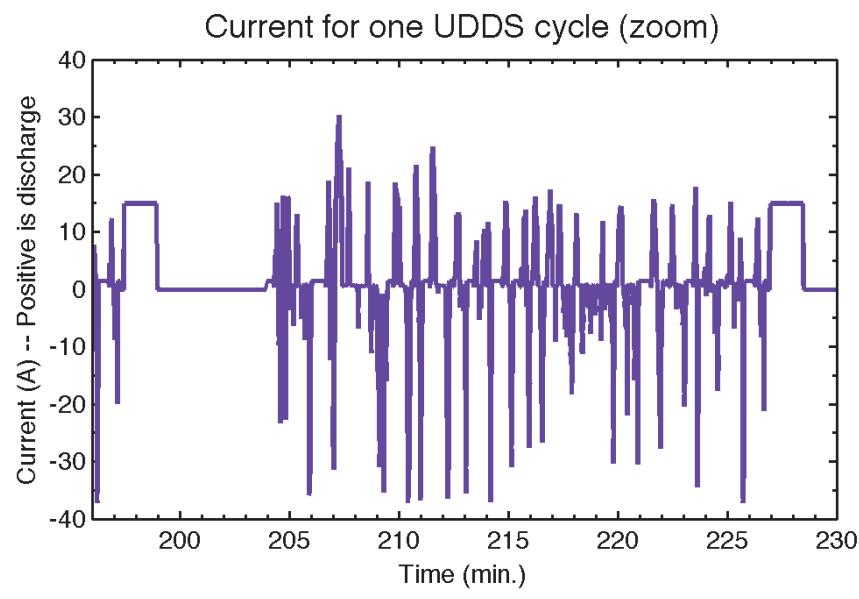

Figure 4. Dynamic test: Current vs. time for one cycle.

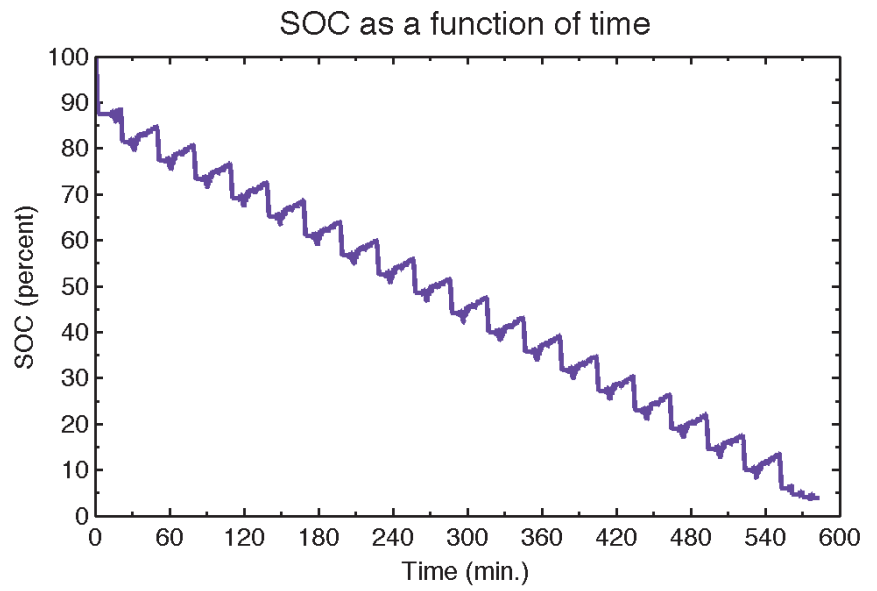

Figure 5. Dynamic test: SOC versus time.

\section{PARAMETER IDENTIFICATION}

The remaining unknown battery parameters could not be directly measured. These parameters were adjusted to best match model simulation results to the UDDS cycle data. Simulink Parameter Estimation was used to simplify and automate the process of identifying these unknown parameters. Simulink Parameter Estimation uses numerical optimization algorithms that estimate parameter values to minimize error between a model's simulated results and measured time-domain data.
Determining the optimum settings for the estimation and simultaneously selecting which parameters to estimate is not a trivial task. Although Simulink Parameter Estimation has knowledge of the model output values that the user provides, it cannot easily account for relationships between the parameters that are being tuned. If multiple parameters being estimated have a similar effect on the output, the optimization algorithms alone cannot distinguish between the similar parameters. Unless a sufficient variety of data is available to exercise all estimated parameters fully, it is typically necessary to take a careful and methodical approach to estimating parameter values.

REDUCING THE PROBLEM COMPLEXITY - The parameters $\mathrm{C}_{\mathrm{n}}, \beta, \mathrm{C}, \mathrm{M}$, and $\mathrm{R}$ needed to be identified. These parameters were temperature-dependent, and lookup tables were used to calculate the parameter values versus temperature, as shown in Figure 6. Assuming eight temperature breakpoints were used, we would have had a total of 40 parameters to tune.

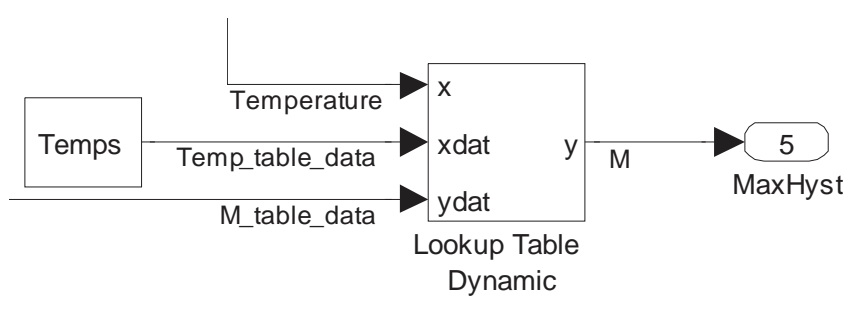

Figure 6. Parameter lookup table.

In Simulink Parameter Estimation, the nonlinear leastsquares algorithm ( 1 sqnon 1 in) requires at least $(2 n+1)$ simulations per iteration, where $n$ is the number of parameters being estimated. As the number of data sets and estimated parameters increased, the required CPU time and memory usage also increased significantly. We attempted to estimate all 40 parameters simultaneously, but quickly ran out of memory on a 32bit platform. Further experimentation revealed that the estimation needed to be reduced to about 15 parameters with the eight UDDS data sets to avoid failing due to the 32-bit platform memory limitation. We could have immediately switched to a 64-bit platform to remove the memory limitation, but we instead attempted to reduce the number of parameters. This helped us to reduce the CPU time and memory required for the estimation task, so that we could more quickly and easily investigate the effects of trying various estimation settings and algorithms.

For the ESC model, no dynamic data was available for electrolyte temperature. Thus, we had to make the assumption that electrolyte temperature variation during the OCV and UDDS cycles was insignificant. This simplified the problem by allowing us to assume a constant temperature for each test. 
The estimation was initially broken down by temperature to reduce the number of parameters estimated simultaneously. The data points for each unique temperature were estimated separately, thus reducing the estimation task to only five parameters at once. Since only one UDDS data set was available for each temperature, only one data set was used for each estimation task. We also attempted to estimate the initial SOC for the UDDS data, although charging techniques were used that ensured that initial SOC for data collection was very close to 1 . The small quantity of parameters and data per estimation greatly improved the estimation speed; an estimation task for each temperature would complete in only a few minutes.

For each temperature, a Simulink Parameter Estimation task was created and run using a MATLAB M-function. Also, only one UDDS data set was used for each estimation task. The following Simulink Parameter Estimation software tool settings were used:

hEst. Optimoptions.Algorithm = 'lsqnonlin';

hEst. Optimoptions. MaxFunEvals = 400;

hEst. Optimoptions. MaxIter $=100$;

hEst. Optimoptions. TolX $=1 e-3$;

hEst. Optimoptions.TolFun $=1 e-3$;

SPECIFICATION OF ESTIMATED PARAMETERS Selection of the optimization algorithm, the estimated parameters, and the parameter constraints for the battery parameterization was a trial-and-error process. In our initial estimation attempt, five parameters were estimated, and their maximum and minimum values were given a broad range. An initial guess for each parameter was chosen based on prior experimentation with the model. Table 1 shows the parameters estimated and their constraints.

\begin{tabular}{|c|c|c|c|}
\hline Parameter & Initial Value & Minimum & Maximum \\
\hline $\mathrm{B}$ & 3 & 0 & 10 \\
\hline $\mathrm{C}$ & -0.5 & -10 & 10 \\
\hline $\mathrm{C}_{\mathrm{n}}$ & 5 & 0 & 10 \\
\hline $\mathrm{R}$ & 0.05 & 0 & 1 \\
\hline $\mathrm{M}$ & 0.01 & 0 & 0.5 \\
\hline
\end{tabular}

Table 1. Parameter constraints: Initial estimation.

The parameters in Table 1 were estimated at each temperature breakpoint using the UDDS data sets. The optimized parameter values were plotted for each temperature to examine parameter sensitivities and determine whether the values were realistic. It was assumed that the battery was fully charged to $100 \%$ SOC at the beginning of each data set.

The optimized parameters in Figure 7 showed some variability in parameters $C_{n}$ (capacity), $C$ (voltage relaxation), and $\mathrm{M}$ (maximum hysteresis). The optimized table values for these parameters did not have smooth relationships with temperature. There are a few possible reasons for this variation. It is possible that there was some inconsistency in the data itself, or in the physical battery. However, it is also possible that there was some linkage between parameters that were tuned, meaning that adjusting two separate parameters may have had a similar effect on the results. Simulink Parameter Estimation cannot distinguish between linked parameters based solely on output data dependent equally on both linked parameters. Assuming this was the case, the optimization problem was not well constrained. We investigated a few possible remedies to further constrain the estimation.

One possible solution would have been to add data sets that better exercised these parameters. Providing measured data under a variety of operating conditions and equalizing the weights between data sets may have been helpful. However, we had limited data to work with so this was not feasible.
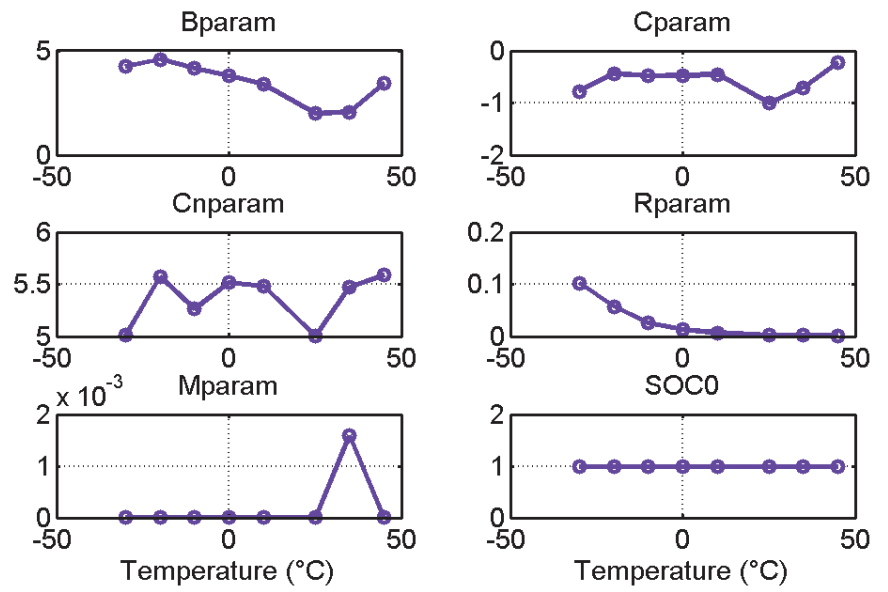

Figure 7. Parameter values: Estimating five parameters.

A second potential solution was to fit characteristic equations to these parameters to constrain the shape of each parameter curve. This method was investigated, but it would have required significant CPU time for the estimation tasks. We did not initially attempt this, because the long estimation times would have hindered our ability to examine different estimation settings.

The third solution was to break up the estimation into steps, to isolate the parameters. In particular, we knew that the battery capacity $C_{n}$ should increase with temperature in the $-30^{\circ} \mathrm{C}$ to $50^{\circ} \mathrm{C}$ range. Using the $\mathrm{O} \mathrm{CV}$ data, we could separately parameterize the capacity parameter $C_{n}$, using capacity optimization techniques ${ }^{[5]}$.

For the ESC model, the capacity model was implemented as a temperature-dependent lookup table. OCV discharge curves at a $\mathrm{C} / 30$ rate were assumed to have a low enough discharge current that the electrolyte temperature was constant and the full capacity of the battery was extracted. The integration of extracted charge for the OCV data at each temperature was used to determine the maximum extracted charge, as shown in Figure 8. 


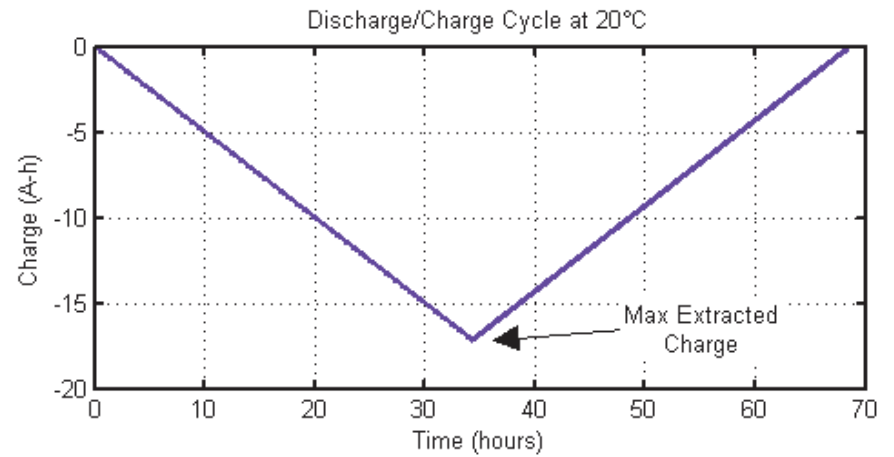

Figure 8. Charge extracted during $20^{\circ} \mathrm{C}$ cycle.

We repeated the estimation, this time using fixed values for $C_{n}$. Table 2 shows the parameter settings, and Figure 9 shows the optimized parameter values.

\begin{tabular}{|c|c|c|c|}
\hline Parameter & Initial Value & Minimum & Maximum \\
\hline B & 3 & 0 & inf \\
\hline C & -0.5 & - inf & inf \\
\hline R & 0.05 & 0 & inf \\
\hline M & 0.01 & 0 & inf \\
\hline
\end{tabular}

Table 2. Parameter constraints: Fixed $C_{n}$.
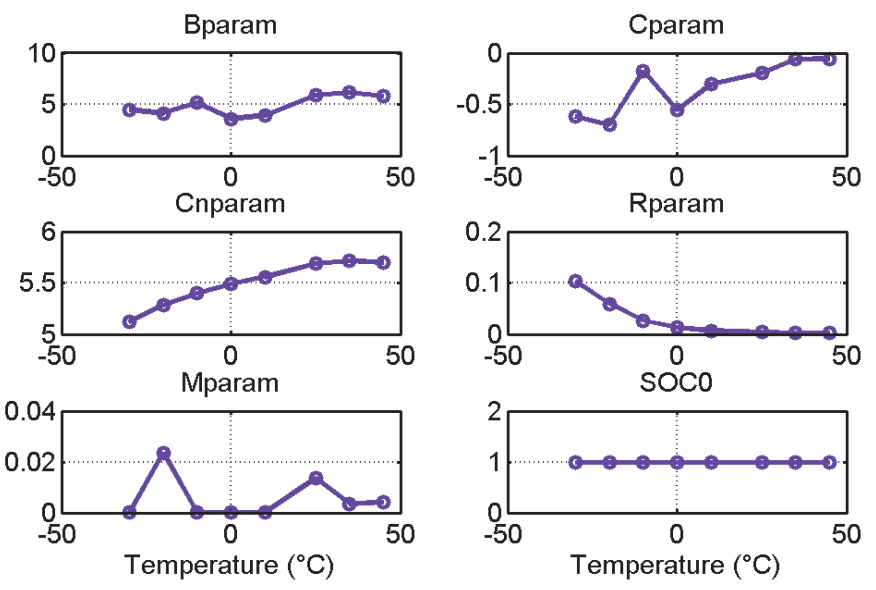

Figure 9. Parameter values: Fixed capacity curve.

In Figure 9, the resulting parameter values did have a slightly smoother relationship with respect to temperature, aside from a few outliers. The relationship for $M$ (hysteresis) was still somewhat inconsistent. It is likely that additional data sets would have better exercised the hysteresis effects, thus allowing us to better parameterize $\mathrm{M}$.

The simulation results for the parameter values in Figure 9 were reasonably accurate across each UDDS data set. Figure 10 shows an example of the simulation results at $10^{\circ} \mathrm{C}$. The accuracy of the simulated volt age versus measured voltage was generally reasonable, but trailed off at the end of the discharge (low SOC).

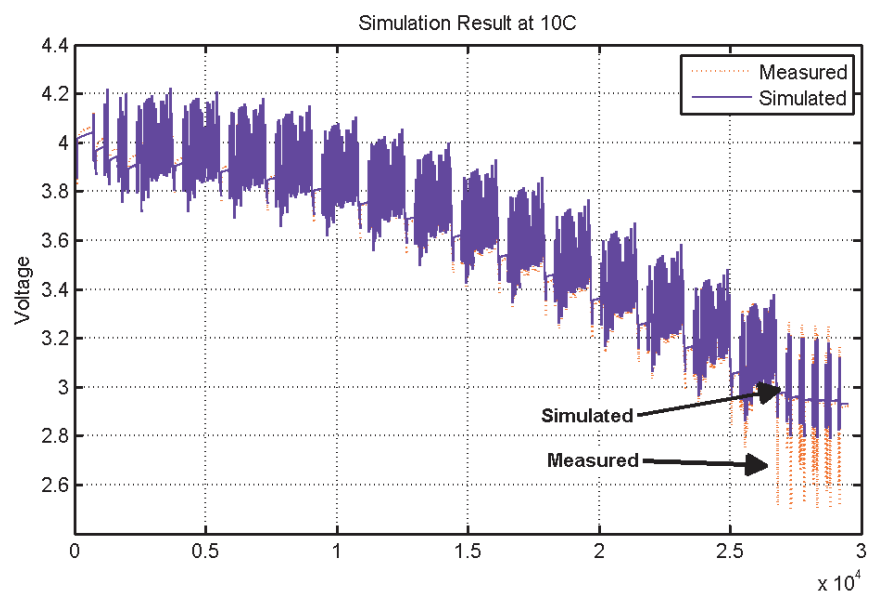

Figure 10 . Simulation results $\left(1^{\circ} \mathrm{C}\right)$.

Figure 11 provides a closer look at the transients in the simulation; the simulated voltage is close to the measured value, but there is some difference in the shape during transient events. The sharp simulated transients could be due to limitations in the model itself or the limited amount of data available.

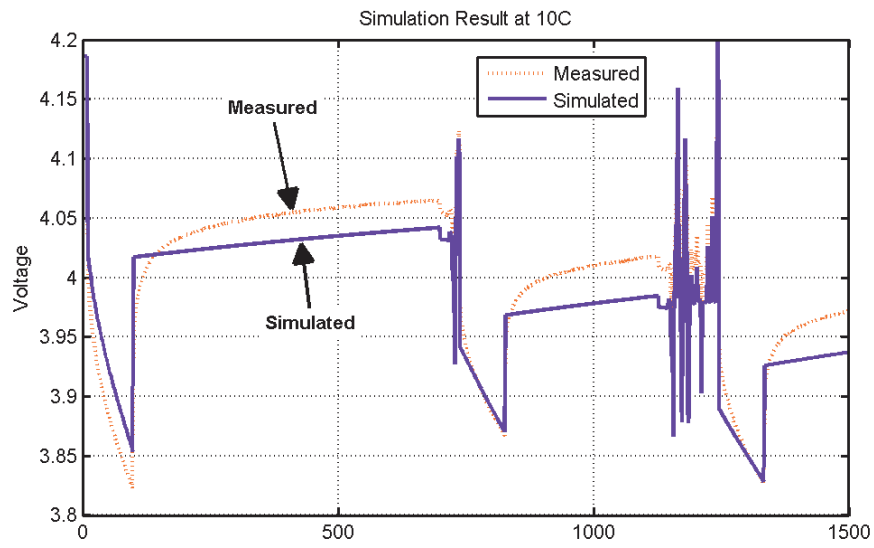

Figure 11. Simulation results zoomed $\left(10^{\circ} \mathrm{C}\right)$.

The RMS error of the simulation data was computed and averaged over time for each UDDS data set (see Figure 12). The maximum observed error in each data set was also plotted. Across all temperatures, the mean voltage error was $0.038 \mathrm{~V}$, or $1 \%$ of nominal cell voltage. The maximum voltage error during simulation varied with each data set and averaged $0.46 \mathrm{~V}$. Although this is a large error, it always occurred at low SOC. 

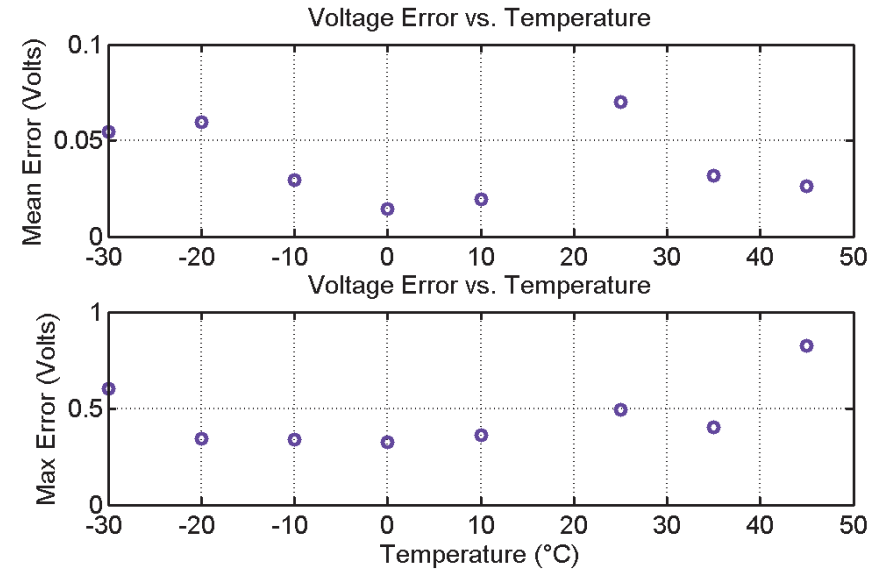

Figure 12. Simulation error (UDDS) vs. temperature.

\section{FUTURE WORK}

Although the Simulink Parameter Estimation parameterization of the ESC battery cell model gave reasonable results, a few areas could benefit from further study:

- The accuracy of the simulation results averaged about $1 \%$ error, but there was still room for improvement, particularly in the shape of the transients and also the response at low SOC.

- The parameter lookup tables were expected to have smooth curves with respect to temperature. Ideally, we would fit equations in place of the tables to help constrain the parameters during estimation and to ensure that the parameters' variance with temperature fit a more realistic shape.

To make these improvements, we would need additional data sets. Ideally, we would like to have a variety of data sets at each temperature, including OCV curves and transient curves like the UDDS data. Additional data sets would give a better overall result, and the parameter curves would likely be smoother.

Improvements to the model itself may also assist in improving the results. The shape of the transient response appeared to need some additional time constant to better match the measured results. Although low SOC modeling was not a concern for HEV applications, the simulation error at low SOC may benefit from a nonlinear SOC-dependent model of resistance (whose value can increase quite dramatically at low SOC). The addition of thermal modeling to the battery may also provide improvement.

Additionally, we would likely need to use a 64-bit platform for the estimations. Ideally we would like to fit curves to each parameter and also add a thermal model to the battery. These changes would require that we estimate all temperatures simultaneously, which would increase the number of parameters estimated and consequently increase the CPU time and memory usage.

\section{CONCLUSION}

This paper describes the development of a lithium polymer battery (LiPB) cell model, along with a process to parameterize the model from measured laboratory data. The construction and simulation of the battery model are described. The paper also documents a process to estimate parameters for the model. In particular, Simulink Parameter Estimation was used with a MATLAB and Simulink based cell model. The optimized battery model simulated a number of UDDS cycles accurately, with $1 \%$ average voltage error.

\section{ACKNOWLEDGMENTS}

This work was supported in part by Compact Power Inc. (CPI). The use of company facilities is gratefully acknowledged.

\section{REFERENCES}

1. Plett, G., "Results of Temperature-Dependent LiPB Cell Modeling for HEV SOC Estimation," CD-ROM Proceedings of the 21st Electric Vehicle Symposium (EVS21) (Monaco: April 2005).

2. Plett, G., "Sigma-Point Kalman Filters for Battery Management Systems of LiPB-Based HEV Battery Packs-Parts 1-2," Journal of Power Sources, 152 (2), (2006) 1356-84.

3. Plett, G., Klein, M., "Advances in HEV Battery Management Systems," CD-ROM Proc. SAE Convergence 2006 (Detroit, MI: October 2006).

4. Plett, G., "Extended Kalman filtering for battery management systems of LiPB-based HEV battery packs-Parts 1-3," Journal of Power Sources 134 (2) (2004) 252-92.

5. Jackey, Robyn A., "A Simple, Effective Lead-Acid Battery Modeling Process for Electrical System Component Selection," SAE Paper 2007-01-0778, SAE International, Warrendale, PA, 2007.

\section{CONTACT}

Robyn Jackey is a technical consultant at The MathWorks. He may be reached at:

The MathWorks

39555 Orchard Hill Place

Suite 280

Novi, MI 48375

E-mail: robyn.jackey@mathworks.com

Gregory Plett is associate professor of electrical engineering at the University of Colorado at Colorado Springs. He may be reached at:

Dept. of Electrical and Computer Engineering 
University of Colorado at Colorado Springs

1420 Austin Bluffs Parkway

P.O. Box 7150

Colorado Springs, CO 80933-7150

Tel: +1-719-262-3468

Fax: +1-719-262-3589

E-mail: glp@eas.uccs.edu

URL: mocha-java.uccs.edu

Martin Klein is director of engineering at Compact Power Inc. He may be reached at:

Compact Power Inc.

1857 Technology Drive

Troy, MI 48083

Tel: +1-248-307-1800

E-mail: mklein@compactpower.com

URL: www.compactpower.com

MATLAB and Simulink are registered trademarks of The MathWorks, Inc.

See www.mathworks.com/trademarks for a list of additional trademarks.

Other product or brand names may be trademarks or registered

trademarks of their respective holders. 